\title{
A weighted full-Newton step primal-dual interior point algorithm for convex quadratic optimization
}

\author{
Mohamed Achache ${ }^{1, *}$ \\ ${ }^{1}$ Laboratoire de Mathématiques Fondamentales et Numériques, Université Ferhat Abbas de Sétif 1
}

Received 6 July 2013; Accepted 15 January 2014

Editor: Junfeng Yang

\begin{abstract}
In this paper, a new weighted short-step primal-dual interior point algorithm for convex quadratic optimization (CQO) problems is presented. The algorithm uses at each interior point iteration only full-Newton steps and the strategy of the central path to obtain an $\epsilon$-approximate solution of CQO. This algorithm yields the best currently wellknown theoretical iteration bound, namely, $O\left(\sqrt{n} \log \frac{n}{\epsilon}\right)$ which is as good as the bound for the linear optimization analogue.
\end{abstract}

Keywords Convex quadratic optimization; weighted interior point methods; short-step primal-dual algorithms; complexity of algorithms

DOI: $10.19139 /$ soic.v2i1.21

\section{Introduction}

Consider the quadratic optimization (QO) problem in standard format:

$$
(P) \min _{x}\left\{c^{T} x+\frac{1}{2} x^{T} Q x: A x=b, x \geq 0\right\}
$$

and its dual problem

$$
\text { (D) } \max _{x, y, z}\left\{b^{T} y-\frac{1}{2} x^{T} Q x: A^{T} y+z-Q x=c, z \geq 0\right\},
$$

\footnotetext{
${ }^{*}$ Correspondence to: Laboratoire de Mathématiques Fondamentales et Numériques. Université Ferhat Abbas de Sétif 1. Algérie. e-mail: achache_m@yahoo.fr
}

ISSN 2310-5070 (online) ISSN 2311-004X (print)

Copyright (c) 2014 International Academic Press 
where $Q$ is a given $(n \times n)$ real symmetric matrix, $A$ is a given $(m \times n)$ real matrix with $\operatorname{rank}(A)=m, c \in \mathbf{R}^{n}, b \in \mathbf{R}^{m}, x \in \mathbf{R}^{n}, z \in \mathbf{R}^{n}$ and $y \in \mathbf{R}^{m}$.

The QO problems have many important applications in optimization and mathematical programming problems.

There are a variety of solution approaches for CQO which have been studied intensively. Among them, the interior-point methods (IPMs) gained more attention than others methods. Feasible primal-dual path-following methods are the most attractive methods of IPMs [7, 9]. Their derived algorithms achieved important results such as polynomial complexity and numerical efficiency. These algorithms trace approximately the so-called central-path which is a curve that lies in the feasible region of the considered problem and they reach an optimal solution of it. However, in practice these methods don't always find a strictly feasible centered point to starting their derived algorithms. So, it is worth analyzing other cases when the starting points are not centered. Thus leads to the concept of Target-Following IPMs introduced by Jansen et al.,[6] as a generalization of the classical path-following methods. These methods are based on the observation that with every algorithm which follows the central-path we associate a target sequence on the central-path. Weighted path-following methods can be viewed as a particular case of it. These methods were studied extensively by many authors $[3,4,5,7,8]$ for Linear optimization (LO) and linear complementarity problem (LCP). Recently, Achache and Khebchache [1], introduced a new weighted method for monotone LCP where the complexity of the corresponding short-step algorithm is $O\left(\sqrt{n} \log \frac{n}{\epsilon}\right)$. Motivated by their work, we propose a new weighted primal-dual path-following algorithm for solving CQO. The algorithm uses at each interior point iteration only weighted full-Newton steps and the strategy of the central path to get an $\epsilon$-approximate solution of CQO. We prove that the shortstep algorithm has the following iteration bound $O\left(\sqrt{n} \log \frac{n}{\epsilon}\right)$ which is as good as the bound for LO [3, 7, 8], CQO [1, 3] and LCP [2, 7], analogue. The algorithm has advantages that no line searches is needed and it can start with a suitable starting point not necessarily centered.

The rest of the paper is built as follows. In Section 2, the weighted-path and the search direction are presented. The generic weighted primal-dual path-following algorithm for CQO is also stated. In Section 3, the analysis of the algorithm and the iteration bound with short-step method are presented. Finally, a conclusion and future remarks follow in Section 4.

The notation used in this paper is as follows. $\mathbf{R}^{n}$ denotes the space of $n$ dimensional real vectors and $\mathbf{R}_{++}^{n}$ is the set of all positive vectors of $\mathbf{R}^{n}$. Given $x, z \in \mathbf{R}_{++}^{n}$, their Hadamard product is $x z=\left(x_{1} z_{1}, \ldots, x_{n} z_{n}\right)^{T}$. The expressions $\|u\|=\sqrt{u^{T} u}$ and $\|u\|_{\infty}=\max _{i}\left|u_{i}\right|$ denote the Euclidean and the maximum norms for a vector $u$, respectively. Let $x, z \in \mathbf{R}_{++}^{n}, \sqrt{x}=$ $\left(\sqrt{x_{1}}, \ldots, \sqrt{x_{n}}\right)^{T}, x^{-1}=\left(x_{1}^{-1}, \ldots, x_{n}^{-1}\right)^{T}$ and $\frac{x}{z}=\left(\frac{x_{1}}{z_{1}}, \ldots, \frac{x_{n}}{z_{n}}\right)^{T}$. Let $g(x)$ and $f(x)$, be two positive real valued functions, then $g(x)=O(f(x)) \Leftrightarrow g(x) \leq$ 
$k f(x)$ for some positive constant $k$. Finally, the vector of all ones and the identity matrix are denoted by $e$ and $I$, respectively.

\section{The weighted-path and the search direction}

Throughout the paper, we make the following assumptions for QO.

Assumption 1. Interior Point Condition (IPC). There exists a triplet of vectors $\left(x^{0}, y^{0}, z^{0}\right)$ such that:

$$
A x^{0}=b, x^{0}>0, A^{T} y+z^{0}-Q x^{0}=c, z^{0}>0 .
$$

Assumption 2. Positive semidefiniteness. The matrix $Q$ is positive semidefinite, i.e., for all $v \in \mathbf{R}^{n}, v^{T} Q v \geq 0$.

Finding an approximate solution of $(P)$ and $(D)$ is equivalent to solving the following system of optimality conditions for $(P)$ and $(D)$ :

$$
\begin{cases}A x & =b, x \geq 0 \\ A^{T} y+z-Q x & =c, z \geq 0 \\ x z & =0\end{cases}
$$

The basic idea behind weighted primal-dual interior-point algorithm is to replace the third equation (complementarity condition) in (1) by the parametrized equation $x z=w$ with $w$ is a positive vector in $\mathbf{R}^{n}$. Thus, we consider the following perturbed system:

$$
\begin{cases}A x & =b, x \geq 0 \\ A^{T} y+z-Q x & =c, z \geq 0 \\ x z & =w\end{cases}
$$

Under Assumption 1 and Assumption 2, the system (2) has a unique solution denoted by $(x(w), y(w), z(w))$ for all $w>0$ [2]. The set

$$
\{(x(w), y(w), z(w)): w>0\}
$$

is called the weighted-path of problems $(P)$ and $(D)$. If $w$ goes to zero, then the limit of the weighted-path exists and since the limit point satisfies the complementarity condition, the limit yields an optimal solution for CQO. This limiting property of the weighted-path leads to the main idea of the iterative primal-dual methods for solving (2).

Remark 2.1

If $w=\mu e$ with $\mu>0$, then the weighted-path coincides with the classical centralpath. 
Now, we proceed to describe a weighted full-Newton step produced by the algorithm for a given $w>0$. Applying Newton's method for (2) for a given feasible point $(x, y, z)$ then the Newton direction $(\Delta x, \Delta y, \Delta z)$ at this point is the unique solution of the following linear system of equations:

$$
\left(\begin{array}{ccc}
A & 0 & 0 \\
-Q & A^{T} & I \\
Z & 0 & X
\end{array}\right)\left(\begin{array}{c}
\Delta x \\
\Delta y \\
\Delta z
\end{array}\right)=\left(\begin{array}{c}
0 \\
0 \\
w-X z
\end{array}\right)
$$

where $X:=\operatorname{diag}(x), Z:=\operatorname{diag}(z)$.

Again under our assumptions and the fact that $\operatorname{rank}(A)=m$, the system (3) has a unique solution $(\Delta x, \Delta y, \Delta z)$. Hence, a new weighted full-Newton iteration is constructed according to:

$$
x_{+}:=x+\Delta x ; y_{+}:=y+\Delta y ; \text { and } z_{+}=z+\Delta z .
$$

To simplify the matters, we define the vectors:

$$
v:=\sqrt{x z} \text { and } d:=\sqrt{x z^{-1}} .
$$

The vector $d$ uses to scale the vectors $x$ and $z$ to the same vector $v$ as

$$
d^{-1} x=d z=v
$$

and as well as for the original directions to the scaling directions:

$$
d_{x}=d^{-1} \Delta x \text { and } d_{z}=d \Delta z .
$$

It follows that:

$$
x \Delta z+z \Delta x=v\left(d_{x}+d_{z}\right)
$$

and

$$
d_{x} d_{z}=\Delta x \Delta z=\Delta x Q \Delta x \geq 0,
$$

since $Q$ is a semidefinite matrix.

Hence, by using (5), (6) and (7), the system (3) becomes:

$$
\left(\begin{array}{lll}
\bar{A} & 0 & 0 \\
-\bar{Q} & \bar{A}^{T} & I \\
I & 0 & I
\end{array}\right)\left(\begin{array}{l}
d_{x} \\
d_{y} \\
d_{z}
\end{array}\right)=\left(\begin{array}{l}
0 \\
0 \\
p_{v}
\end{array}\right)
$$

where

$$
p_{v}=v^{-1}\left(w-v^{2}\right)
$$

and $\bar{A}=D A D$ and $\bar{Q}=D Q D$ with $D:=\operatorname{diag}(d)$.

In the next sub-section, we describe the generic feasible weighted primal-dual path-following algorithm to solve CQO. 


\subsection{The Algorithm}

Similar to LO case, we define for any positive vector $v$ and in view of (9), a normbased proximity measure as follows:

$$
\delta(v ; w)=\frac{\left\|p_{v}\right\|}{2 \sqrt{\min (w)}}=\frac{\left\|v^{-1}\left(w-v^{2}\right)\right\|}{2 \sqrt{\min (w)}} .
$$

One can easily verify that

$$
\delta(v ; w)=0 \Leftrightarrow v^{2}=w \Leftrightarrow x z=w .
$$

Hence the value $\delta(v ; w)$ is to measure the distance of a point $(x, y, z)$ to the weighted-path $(x(w), y(w), z(w))$.

Let denote another measure $\sigma_{C}(w)$ as follows

$$
\sigma_{C}(w)=\frac{\max (w)}{\min (w)} .
$$

The role of $\sigma_{C}(w)$ is to measure the closeness of $w$ to the central path.

Here,

$$
\min (w)=\min _{i}\left(w_{i}\right)
$$

and likewise

$$
\max (w)=\max _{i}\left(w_{i}\right) .
$$

Note that in (11), $\sigma_{C}(w) \geq 1$, with equality if $w$ is on the central-path.

Now we are ready to describe the generic weighted path-following interior-point algorithm for CQO as follows.

A generic weighted Primal-Dual Path-Following Algorithm for CQO

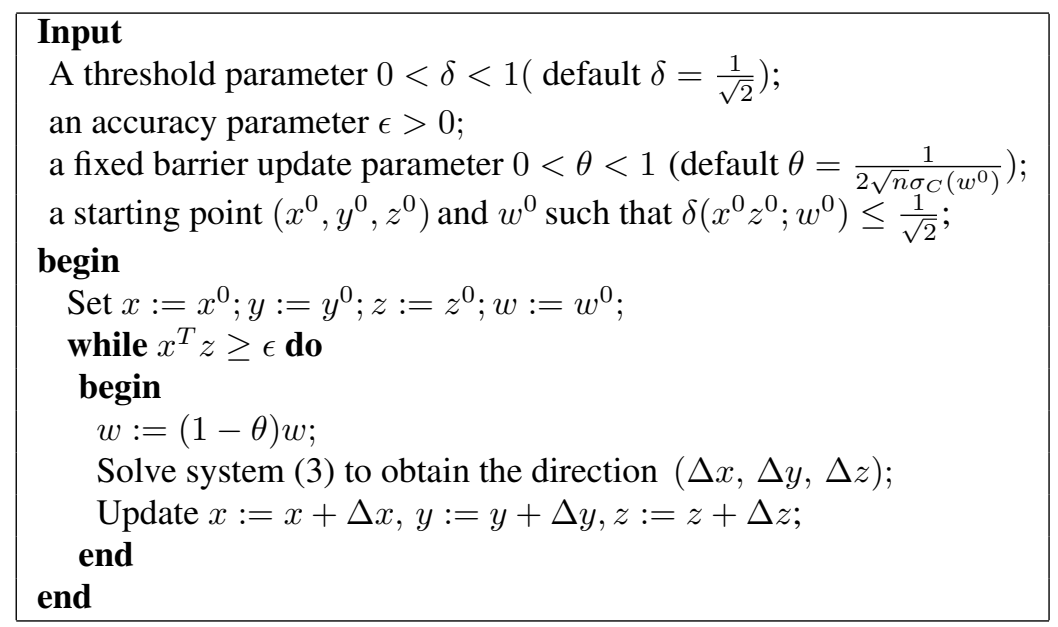


Algorithm 2.1

In the next section, we will show that Algorithm 2.1 is well-defined for the defaults $\theta=\frac{1}{2 \sqrt{n} \sigma_{C}\left(w^{0}\right)}$ and $\delta \leq \frac{1}{\sqrt{2}}$ and can solve CQO in polynomial-time.

\section{Complexity analysis}

In the next lemma, we state some useful technical results that will be used later in the analysis of the algorithm.

\section{Lemma 3.1}

Let $\left(d_{x}, d_{z}\right)$ be a solution of (8) and suppose $w>0$. If $\delta:=\delta(v ; w)$. Then, one has

$$
0 \leq d_{x}^{T} d_{z} \leq 2 \delta^{2} \min (w),
$$

and

$$
\left\|d_{x} d_{z}\right\|_{\infty} \leq \delta^{2} \min (w) \text { and }\left\|d_{x} d_{z}\right\| \leq \sqrt{2} \delta^{2} \min (w) .
$$

Proof: Since $0 \leq d_{x}^{T} d_{z}$, the statement in (12) follows immediately from the following equality:

$$
\left\|d_{x}\right\|^{2}+\left\|d_{z}\right\|^{2}+2 d_{x}^{T} d_{z}=\left\|d_{x}+d_{z}\right\|^{2}=\left\|p_{v}\right\|^{2}=4 \delta^{2} \min (w) .
$$

For (13), (see Lemma C.4 in [7]), since

$$
\left\|d_{x} d_{z}\right\|_{\infty} \leq \frac{1}{4}\left\|p_{v}\right\|^{2} \text { and }\left\|d_{x} d_{z}\right\| \leq \frac{1}{2 \sqrt{2}}\left\|p_{v}\right\|^{2} .
$$

This completes the proof.

The following lemma shows that the feasibility of the weighted full-Newton step under the condition $\delta:=\delta(v ; w)<1$.

\section{Lemma 3.2}

Let $(x, z)$ be a strictly feasible primal-dual point. Then $x_{+}=x+\Delta x>0$ and $z_{+}=y+\Delta z>0$ if and only if $w+d_{x} d_{z}>0$.

Proof: For the first statement we have,

$$
\begin{aligned}
x_{+} z_{+} & =(x+\Delta x)(z+\Delta z) \\
& =x z+x \Delta z+z \Delta x+\Delta x \Delta z \\
& =x z+(w-x z)+\Delta x \Delta z \\
& =w+\Delta x \Delta z .
\end{aligned}
$$

Then from equation in (7), we have,

$$
\begin{aligned}
x_{+} z_{+} & =w+\Delta x \Delta z \\
& =w+d_{x} d_{z} .
\end{aligned}
$$


If the full-Newton step is strictly feasible $x_{+}>0$ and $z_{+}>0$ then $x_{+} z_{+}>0$ and so $w+d_{x} d_{z}>0$.

To show that $x_{+}$and $z_{+}$are positive, we introduce a step length $\alpha \in[0,1]$ and we define

$$
x^{\alpha}=x+\alpha \Delta x, \quad z^{\alpha}=z+\alpha \Delta z .
$$

So $x^{0}=x, x^{1}=x_{+}$and similar notations for $z$, hence $x^{0} z^{0}=x z>0$. We have,

$$
x^{\alpha} z^{\alpha}=(x+\alpha \Delta x)(z+\alpha \Delta z)=x z+\alpha(x \Delta z+z \Delta x)+\alpha^{2} \Delta x \Delta z .
$$

Now by using (6), we get

$$
x^{\alpha} z^{\alpha}=x z+\alpha(w-x z)+\alpha^{2} \Delta x \Delta z .
$$

We assume that $w+d_{x} d_{z}>0$, we deduce that $w+\Delta x \Delta z>0$ which equivalent to $\Delta x \Delta z>-w$. Substitution we obtain

$$
\begin{aligned}
x^{\alpha} z^{\alpha} & >x z+\alpha(w-x z)-\alpha^{2} w \\
& =(1-\alpha) x z+\left(\alpha-\alpha^{2}\right) w \\
& =(1-\alpha) x z+\alpha(1-\alpha) w .
\end{aligned}
$$

Since $x z$ and $w$ are positive it follows that $x^{\alpha} z^{\alpha}>0$ for $\alpha \in[0,1]$. Hence, none of the entries of $x^{\alpha}$ and $z^{\alpha}$ vanish for $\alpha \in[0,1]$. Since $x^{0}$ and $z^{0}$ are positive, this implies that $x^{\alpha}>0$ and $z^{\alpha}>0$ for $\alpha \in[0,1]$. Hence, by continuity argument, the vectors $x^{\alpha}$ and $z^{\alpha}$ must be positive which proves that $x_{+}$and $z_{+}$are positive. This completes the proof.

\section{Lemma 3.3}

If $\delta:=\delta(v ; w)<1$. Then, the primal-dual full-Newton step is strictly feasible,i.e., $x_{+}>0$ and $z_{+}>0$.

Proof: In Lemma 3.2, we have seen that:

$$
x_{+} z_{+}>0 \text { if } w+d_{x} d_{z}>0 .
$$

So $w+d_{x} d_{z}>0$ holds if

$$
w_{i}+\left(d_{x}\right)_{i}\left(d_{z}\right)_{i}>0, \text { for all } i .
$$

We have

$$
w_{i}+\left(d_{x}\right)_{i}\left(d_{z}\right)_{i} \geq w_{i}-\left|\left(d_{x}\right)_{i}\left(d_{z}\right)_{i}\right| \geq \min (w)-\left\|d_{x} d_{z}\right\|_{\infty} \text { for all } i .
$$

Now, according to (13), Lemma 3.1, it follows that:

$$
\min (w)-\left\|d_{x} d_{z}\right\|_{\infty}>\min (w)\left(1-\delta^{2}\right) .
$$


Thus $w+d_{x} d_{z}>0$ holds if $\delta<1$. This completes the proof.

For convenience, we may write

$$
v_{+}=\sqrt{x_{+} z_{+}} .
$$

Lemma 3.4

If $\delta<1$. Then

$$
\left\|v_{+}^{-1}\right\| \leq \frac{1}{\sqrt{\min (w)\left(1-\delta^{2}\right)}} .
$$

Proof: It follows straightforwardly from Lemma 3.3 and since

$$
v_{+}^{-2}=\frac{e}{w+d_{x} d_{z}} .
$$

In the next lemma, we show the influence of a weighted full-Newton step on the proximity measure.

\section{Lemma 3.5}

If $\delta<1$. Then

$$
\delta_{+}:=\delta\left(v_{+} ; w\right) \leq \frac{\delta^{2}}{\sqrt{2\left(1-\delta^{2}\right)}} .
$$

Proof: By definition, we have,

$$
\begin{aligned}
\delta_{+} & =\frac{1}{2 \sqrt{\min (w)}}\left\|v_{+}^{-1}\left(w-v_{+}^{2}\right)\right\| \\
& \leq \frac{1}{2 \sqrt{\min (w)}}\left\|v_{+}^{-1}\right\|\left\|w-v_{+}^{2}\right\| .
\end{aligned}
$$

But $w-v_{+}^{2}=-d_{x} d_{z}$ and $v_{+}^{-1}=\frac{e}{\sqrt{w+d_{x} d_{z}}}$, then by Lemmas 3.1 and 3.4, we have,

$$
\begin{aligned}
\delta_{+} & =\frac{1}{2 \sqrt{\min (w)}}\left\|\frac{d_{x} d_{z}}{\sqrt{w+d_{x} d_{z}}}\right\| \\
& =\frac{1}{2 \sqrt{\min (w)}} \frac{\left\|d_{x} d_{z}\right\|}{\left\|\sqrt{w+d_{x} d_{z}}\right\|} \\
& \leq \frac{1}{2 \sqrt{\min (w)}} \frac{\sqrt{2} \min (w) \delta^{2}}{\sqrt{\min (w)-\left\|d_{x} d_{z}\right\|_{\infty}}} \\
& \leq \frac{1}{2 \sqrt{\min (w)}} \frac{\sqrt{2} \min (w) \delta^{2}}{\sqrt{\min (w)\left(1-\delta^{2}\right)}} \\
& \leq \frac{\delta^{2}}{\sqrt{2\left(1-\delta^{2}\right)}} .
\end{aligned}
$$

This completes the proof. 
Corollary 3.1

If $\delta<1$. Then $\delta_{+} \leq \delta^{2}$ which indicates the convergence quadratic of the proximity when iterations are closed to the path. In addition if $\delta \leq \frac{1}{\sqrt{2}}$, then $\delta_{+} \leq \frac{1}{2}$.

In the next lemma, we discuss the influence on the proximity measure of the update barrier parameter $w_{+}=(1-\theta) w$ on the Newton process along the weighted-path.

Lemma 3.6

If $\delta(w ; v)<1$ and $w_{+}=(1-\theta) w$ where $0<\theta<1$. Then

$$
\delta\left(v_{+} ; w_{+}\right) \leq \frac{\theta}{2 \sqrt{1-\theta} \sqrt{1-\delta^{2}}} \sqrt{n} \sigma_{C}(w)+\frac{1}{\sqrt{2(1-\theta)}} \delta_{+} .
$$

In addition, if $\delta \leq \frac{1}{\sqrt{2}}, \theta=\frac{1}{2 \sqrt{n} \sigma_{C}(w)}$ and $n \geq 3$, then we have,

$$
\delta\left(v_{+} ; w_{+}\right) \leq \frac{1}{\sqrt{2}} .
$$

Proof: Let $\delta\left(v_{+} ; w_{+}\right)$and $w_{+}=(1-\theta) w$ with $0<\theta<1$. Then, by definition we have,

$$
\begin{aligned}
\delta\left(v_{+} ; w_{+}\right) & =\frac{1}{2 \sqrt{\min \left(w_{+}\right)}}\left\|v_{+}^{-1}\left(w_{+}-v_{+}^{2}\right)\right\| \\
& =\frac{1}{2 \sqrt{1-\theta} \sqrt{\min (w)}}\left\|v_{+}^{-1}\left(w_{+}-v_{+}^{2}\right)\right\| \\
& =\frac{1}{2 \sqrt{1-\theta} \sqrt{\min (w)}}\left\|v_{+}^{-1}\left(w_{+}-w+w-v_{+}^{2}\right)\right\| \\
& \leq \frac{1}{2 \sqrt{1-\theta} \sqrt{\min (w)}}\left(\left\|v_{+}^{-1}\right\|\left(\left\|w_{+}-w\right\|+\left\|w-v_{+}^{2}\right\|\right)\right) .
\end{aligned}
$$

Now since $w-v_{+}^{2}=-d_{x} d_{z}$ and $w_{+}-w=-\theta w$ and by Lemmas 3.1 and 3.4 and with the fact that $\|w\| \leq \sqrt{n}\|w\|_{\infty}$, we get,

$$
\begin{aligned}
\delta\left(v_{+} ; w_{+}\right) & \leq \frac{1}{2 \sqrt{1-\theta} \min (w) \sqrt{1-\delta^{2}}}\left[\|\theta w\|+\left\|d_{x} d_{z}\right\|\right] \\
& \leq \frac{1}{2 \sqrt{1-\theta} \min (w) \sqrt{1-\delta^{2}}}\left[\|\theta w\|+\min (w) \delta^{2}\right] \\
& \leq \frac{\theta\|w\|}{2 \sqrt{1-\theta} \min (w) \sqrt{1-\delta^{2}}}+\frac{\delta^{2}}{2 \sqrt{1-\theta} \sqrt{1-\delta^{2}}} \\
& \leq \frac{\theta \sqrt{n}\|w\|_{\infty}}{2 \sqrt{1-\theta} \min (w) \sqrt{1-\delta^{2}}}+\frac{\delta^{2}}{2 \sqrt{1-\theta} \sqrt{1-\delta^{2}}} \\
& =\frac{\theta \sqrt{n} \max (w)}{2 \sqrt{1-\theta} \min (w) \sqrt{1-\delta^{2}}}+\frac{\delta^{2}}{2 \sqrt{1-\theta} \sqrt{1-\delta^{2}}} .
\end{aligned}
$$

Stat., Optim. Inf. Comput. Vol. 2, March 2014. 
Using Lemma 3.5 and (11), we have,

$$
\delta\left(v_{+} ; w_{+}\right) \leq \frac{\theta \sqrt{n} \sigma_{C}(w)}{2 \sqrt{1-\theta} \sqrt{1-\delta^{2}}}+\frac{\delta_{+}}{\sqrt{2(1-\theta)}} .
$$

If $\theta=\frac{1}{2 \sqrt{n} \sigma_{C}(w)}$, and observe that $\sigma_{C}(w) \geq 1$, and for $n \geq 3$, then $\theta \leq$ $\frac{1}{4}$. Furthermore, if $\delta \leq \frac{1}{\sqrt{2}}$, then from Corollary 3.1, $\delta_{+} \leq \frac{1}{2}$. Finally, the above inequalities yield $\delta\left(v_{+} ; w_{+}\right) \leq \frac{1}{\sqrt{2}}$. This completes the proof.

Note that, in all the iterates produced by Algorithm 2.1, we have $\sigma_{C}(w)=$ $\sigma_{C}\left(w^{0}\right)$. Thus, we deduce from Lemma 3.6 that for the default $\theta=\frac{1}{2 \sqrt{n} \sigma_{C}\left(w^{0}\right)}$, the conditions $x, y>0$ and $\delta\left(v_{+} ; w_{+}\right) \leq \frac{1}{\sqrt{2}}$ are maintained during the algorithm. Thus, confirms that Algorithm 2.1, is well-defined.

The upper bound of the duality gap after a weighted full-Newton step is presented in the following lemma.

\section{Lemma 3.7}

Let $\delta:=\delta(v ; w) \leq \frac{1}{\sqrt{2}}$ and $x_{+}=x+\Delta x$ and $z_{+}=z+\Delta z$. Then the duality gap satisfies:

$$
x_{+}^{T} z_{+} \leq(n+1) \max (w) .
$$

Proof: By Lemma 3.2, we have seen that

$$
x_{+} z_{+}=w+d_{x} d_{z}
$$

Hence

$$
\begin{aligned}
e^{T}\left(x_{+} z_{+}\right) & =e^{T} w+e^{T} d_{x} d_{z} \\
& =e^{T} w+d_{x}^{T} d_{z} .
\end{aligned}
$$

According to (13), Lemma 3.1 and $\delta \leq \frac{1}{\sqrt{2}}$, we deduce that:

$$
\begin{aligned}
x_{+}^{T} z_{+} & \leq e^{T} w+2 \delta^{2} \min (w), \\
& \leq e^{T} w+\min (w) .
\end{aligned}
$$

Now, since $e^{T} w \leq n \max (w)$, we get

$$
x_{+}^{T} z_{+} \leq(n+1) \max (w) .
$$

This completes the proof.

The following lemma gives an upper bound for the total number of iterations produced by Algorithm 2.1. 


\section{Lemma 3.8}

Let $x^{k+1}$ and $z^{k+1}$ be the $(k+1)-t h$ iteration produced by the Algorithm 2.1, with $w:=w^{k}$.Then

$$
\left(x^{k+1}\right)^{T} z^{k+1} \leq \epsilon
$$

if

$$
k \geq\left[\frac{1}{\theta} \log \frac{2 n \max \left(w^{0}\right)}{\epsilon}\right] .
$$

Proof: By Lemma 3.7, it follows that:

$$
\left(x^{k+1}\right)^{T} z^{k+1} \leq(n+1) \max \left(w^{k}\right)
$$

with

$$
w^{k}=(1-\theta) w^{k-1}=(1-\theta)^{k} w^{0} .
$$

Then, we have

$$
\left(x^{k+1}\right)^{T} z^{k+1} \leq(1-\theta)^{k}(n+1) \max \left(w^{0}\right) \leq(1-\theta)^{k} 2 n \max \left(w^{0}\right),
$$

since $n+1 \leq 2 n$ for all $n \geq 1$.

Thus the inequality $\left(x^{k+1}\right)^{T} z^{k+1} \leq \epsilon$ is satisfied if

$$
(1-\theta)^{k} 2 n \max \left(w^{0}\right) \leq \epsilon .
$$

Now taking logarithms, we may write

$$
k \log (1-\theta) \leq \log \epsilon-\log 2 n \max \left(w^{0}\right)
$$

and since $-\log (1-\theta) \geq \theta$ for $0<\theta<1$, then the inequality holds if

$$
k \theta \geq \log \frac{2 n \max \left(w^{0}\right)}{\epsilon} .
$$

This completes the proof.

Theorem 3.1

Suppose that $x^{0}$ and $z^{0}$ are strictly feasible starting point for CQO, $w^{0}=$ $\frac{x^{0} z^{0}}{2 \max \left(x^{0} z^{0}\right)}$, and such that $\delta\left(x^{0} z^{0} ; w^{0}\right) \leq \frac{1}{\sqrt{2}}$ for $n \geq 3$. If $\theta=\frac{1}{2 \sqrt{n} \sigma_{C}\left(w^{0}\right)}$ then, Algorithm 2.1, requires at most $O\left(\sqrt{n} \sigma_{C}\left(w^{0}\right) \log \frac{n}{\epsilon}\right)$ iterations to obtain an $\epsilon$ approximate solution of CQO.

In particular, if $w^{0}=\frac{1}{2} e$, then Algorithm 2.1, requires at most $O\left(\sqrt{n} \log \frac{n}{\epsilon}\right)$ iterations which is the currently best known iteration bound for short-update methods.

Proof: By taking the value of $\theta$ and $w^{0}$ in Lemma 3.8, the result follows straightforwardly. This completes the proof. 


\section{Conclusion and future remarks}

In this paper, we have presented a weighted full-Newton step path-following method for CQO. At each interior point iteration, only full-Newton steps are used. The favorable polynomial complexity bound for the algorithm with short-step method is deserved, namely, $O\left(\sqrt{n} \log \frac{n}{\epsilon}\right)$ which is as good as LO case. Finally, the numerical implementation of this algorithm remains to be investigated.

\section{REFERENCES}

1. M. Achache and R. Khebchache: A full-Newton step feasible weighted primal-dual interior point algorithm for monotone LCP. Afrika Matematika. Online (2013).

2. M. Achache: A new primal-dual path-following method for convex quadratic programming. Comput. Appl. Math., 25:(2006), pp.97-110.

3. M. Achache: A weighted path-following method for the linear complementarity problem. Universitatis Babes. Bolyai. Series Informatica (49) (1): (2004), pp.61-73.

4. Zs. Darvay: A weighted-path-following method for linear optimization. Studia Univ. BabesBolyai, Informatica. Vol XLVII. N 2. (2002).

5. J. Ding and T.Y. Li: An algorithm based on weighted logarithmic barrier functions for linear complementarity problems. Arabian Journal for Science and Engineering,15 (4): (1990), pp. 679-685.

6. B. Jansen, C. Roos, T. Terlaky and J.Ph. Vial: Primal-dual target-following algorithms for linear programming. Report 93-107. Delft University of Technology. (1993)

7. C. Roos, T. Terlaky, and J.Ph. Vial: Theory and algorithms for linear optimization. An interior point approach. John-Wiley and Sons, Chichester, UK. (1997).

8. G.Q. Wang, Y. J. Yue, and X.Z. Cai: A weighted path-following method for monotone horizontal linear complementarity problem. Fuzzy information and engineering. 54 (2009), pp.479-487.

9. S.J. Wright: Primal-dual interior point methods. Copyright by SIAM. (1997). 